\title{
Impact of mapped EQ-5D utilities on cost-effectiveness analysis: in the case of dialysis treatments
}

\author{
Fan Yang ${ }^{1}\left(\mathbb{D} \cdot\right.$ Nancy Devlin $^{2} \cdot$ Nan $^{L_{u o}}{ }^{3}$
}

Received: 21 March 2018 / Accepted: 6 June 2018 / Published online: 14 June 2018

(c) The Author(s) 2018

\begin{abstract}
Objectives This study aimed to evaluate the performance of EQ-5D data mapped from SF-12 in terms of estimating cost effectiveness in cost-utility analysis (CUA). The comparability of SF-6D (derived from SF-12) was also assessed.

Methods Incremental quality-adjusted life years (QALYs) and incremental cost-effectiveness ratios (ICERs) were calculated based on two Markov models assessing the cost effectiveness of haemodialysis (HD) and peritoneal dialysis (PD) using utility values based on EQ-5D-5L, EQ-5D using three direct-mapping algorithms and two response-mapping algorithms (mEQ-5D), and SF-6D. Bootstrap method was used to estimate the 95\% confidence interval (percentile method) of incremental QALYs and ICERs with 1000 replications for the utilities.

Results In both models, compared to the observed EQ-5D values, mEQ-5D values expressed much lower incremental QALYs (range -14.9 to $-33.2 \%$ ) and much higher ICERs (range 17.5 to $49.7 \%$ ). SF-6D also estimated lower incremental QALYs $(-29.0$ and $-14.9 \%)$ and higher ICERs (40.9 and 17.5\%) than did the observed EQ-5D. The 95\% confidence interval of incremental QALYs and ICERs confirmed the lower incremental QALYs and higher ICERs estimated using mEQ-5D and SF-6D. Conclusion Compared to observed EQ-5D, EQ-5D mapped from SF-12 and SF-6D would under-estimate the QALYs gained in cost-utility analysis and thus lead to higher ICERs. It would be more sensible to conduct CUA studies using directly collected EQ-5D data and to designate one single preference-based measure as reference case in a jurisdiction to achieve consistency in healthcare decision-making.
\end{abstract}

Keywords Cost-effectiveness $\cdot$ Dialysis $\cdot$ EQ-5D $\cdot$ Mapping $\cdot$ SF-6D

JEL Classification I10

\section{Introduction}

Estimation of health utility and quality-adjusted life years (QALYs) is an important part of cost-utility analysis (CUA) in economic evaluation [1]. Health utility can be generated

Electronic supplementary material The online version of this article (https://doi.org/10.1007/s10198-018-0987-x) contains supplementary material, which is available to authorized users.

Fan Yang

fan.bella.yang@york.ac.uk

1 Centre for Health Economics, University of York, Heslington, York YO10 5DD, UK

2 Office of Health Economics, London, UK

3 Saw Swee Hock School of Public Health, National University of Singapore, Singapore, Singapore from several preference-based utility measures, of which the EuroQol 5-dimension (EQ-5D) is the most commonly used. It is the preferred instrument of National Institute for Health and Care Excellence (NICE) in England for QALY estimation [2] and also being recommended or accepted by health technology assessment (HTA) agencies of many other countries [3-5]. Where desirable utility data were not collected, there is a need to map EQ-5D data from other patient-reported outcome instruments [6]. The mapping approach has been endorsed by NICE [7] for use in cases where EQ-5D data are not available and is increasingly popular for the purpose of estimating QALYs in CUAs [6].

There are various mapping algorithms available. The "source" predictive measures used to map to EQ-5D could be condition-specific quality of life measures (such as EORTC QLQ-C30 for cancer patients [8]), generic quality of life measures (such as Short Form 12-item (SF-12) [9]), 
clinical indicators of disease severity (such as Psoriasis Area and Severity Index [10]), or a combination of these. Also, data can be mapped to either the EQ-5D utility values or the EQ-5D item responses [11]. There are currently no clear guidelines on the best mapping method to EQ-5D for QALY estimation; so when deciding which mapping algorithm should be used in a particular study, whether it could generate comparable utility and cost-effectiveness estimates as the primarily collected EQ-5D would be the main consideration.

In addition, where a HTA agency has not stated its recommendation for one preference-based measure, other valid and robust preference-based instruments might be acceptable. Similar to EQ-5D, Short Form 6-dimension (SF-6D) is also widely used to estimate health utility for calculating QALYs [12]. Great differences in utility estimates derived from SF-6D and EQ-5D have been shown to exist [13-15], but only few studies have examined whether SF-6D could lead to comparable cost-effectiveness estimates as the directly derived EQ-5D [16, 17].

Therefore, this study aimed to evaluate the performance of EQ-5D data derived from multiple mapping algorithms in terms of estimating QALY gains in CUAs. The evaluation was based on one cost-utility analysis study of haemodialysis (HD) and peritoneal dialysis (PD) for patients with end-stage renal disease (ESRD) [18]. The comparability of SF-6D was also assessed.

\section{Methods}

\section{Decision analytic models}

Two Markov models used in a previous CUA study [18] were re-run in the present study (see Supplementary Fig. 1 for model structure). Model 1 and model 2 were constructed for non-diabetic and diabetic patients separately using different parameter values based on Singaporean local data and a 10-year time horizon was used (see Supplementary Table 1 for model transition probabilities). The analysis took the societal perspective and costs were reported in 2015 Singapore dollars (\$). Details were reported elsewhere [18].

\section{Quality of life data}

A consecutive sample of 75 patients undergoing HD and 75 patients undergoing PD for at least 3 months were interviewed in a cross-sectional survey using a battery of questionnaires including 5-level EQ-5D (EQ-5D-5L), SF-12, disease-specific scales of the 36-item Kidney Disease Quality of Life questionnaire (KDQOL-36), and questions assessing socio-demographic characteristics [19].

The EQ-5D-5L self-report questionnaire has five items (mobility, self-care, usual activities, pain/discomfort, and anxiety/depression) [20], with five descriptive levels for each item. The five levels include "no problems", "slight problems", "moderate problems", and "severe problems" for all five items, and "unable to do" for mobility, self-care and usual activities and "extreme problems" for pain/discomfort and anxiety/depression. EQ-5D-5L items assess respondents' health status on the day of survey. The SF-12 is a commonly used generic health instrument including 12 items, with a 4-week recall period, producing two summary scores, physical component summary (PCS) and mental component summary (MCS) [21].

\section{Estimation of utilities}

Individual-level utilities were generated through the following approaches. First, utilities were calculated from EQ5D-5L data using the recently developed EQ-5D-5L value set in England [22]. Second, five mapping functions were used to generate EQ-5D values from SF-12, including three functions mapping directly to utility values $[9,23,24]$ and two functions mapping to EQ-5D responses [11]. Mapping function a. was developed using data from a low-income and predominantly minority patient sample attending a community health centre in US while other four functions were developed using the EQ-5D and SF-12 data collected from a representative general population sample in US. The UK EQ-5D-3L value set was used in all functions. Ordinary least squares (OLS) regression equations were used to directly map SF-12 to EQ-5D values including adjusted PCS and MCS (centered on the sample mean) and their interaction terms in function a. [23], PCS, MCS, and their interaction terms in function b [9]. and PCS and MCS only in function c. [24]. Multinomial logit regressions were used to map SF-12 summary scores (function d.) and individuals SF-12 questions (function e.) onto EQ-5D responses, respectively [11]. The mapping-derived utilities are hereafter referred to as "mEQ-5D" values. Third, SF-6D values were generated using responses to seven of the SF-12 items and a recommended algorithm [25], which is based on a set of preference weights obtained from a sample of the general population in the UK. The main characteristics of these methods are summarised in Table 1.

Using these individual-level utility values, multivariate linear regression models were run to predict the mean utility values for HD- and PD-treated non-diabetic and diabetic ESRD patients controlling for socio-demographic characteristics. The predicted mean utility values for HD and PD states were used in model 1 and model 2. Utility values for transplantation were obtained from a published meta-analysis [26]. Utilities values for all health states were assumed constant within the time horizon of both models. 
Table 1 Methods to generate health utility values from the EQ-5D-5L and SF-12 surveys

\begin{tabular}{|c|c|c|c|c|c|}
\hline Calculation methods & Author & Value set country & Sample size & Valuation method & Value range \\
\hline EQ-5D-5L value set & Devlin et al. & England & 912 & Composite TTO \& DCE & $(-0.285,1)$ \\
\hline \multirow[t]{5}{*}{ SF-12 mapped EQ-5D-3L } & Franks et al. & UK & 240 & Direct mapping $^{\mathrm{a}}$ & $(-0.140,0.930)$ \\
\hline & Franks et al. & UK & 12,988 & Direct mapping ${ }^{\mathrm{b}}$ & $(-0.118,0.980)$ \\
\hline & Lawrence and Fleishman & UK & 14,580 & Direct mapping $^{\mathrm{c}}$ & $(-0.131,1)$ \\
\hline & Gray et al. & UK & 12,967 & Response mapping ${ }^{\mathrm{d}}$ & $(-0.594,1)$ \\
\hline & Gray et al. & UK & 12,967 & Response mapping ${ }^{\mathrm{e}}$ & $(-0.594,1)$ \\
\hline SF-12 based SF-6D & Brazier and Roberts & UK & 611 & Standard gamble & $(0.345,1)$ \\
\hline
\end{tabular}

$E Q-5 D$-3L 3-level EuroQol-5D, $S F-12$ Short Form-12, $S F$-6D Short Form 6-dimension, TTO time trade-off, $D C E$ discrete choice experiment ${ }^{a} \mathrm{PCS}$ and MCS were centered on the sample mean and then included in ordinary least squares model with the interaction terms

${ }^{b} \mathrm{PCS}, \mathrm{MCS}$, and the interaction terms were included in ordinary least squares model

${ }^{\mathrm{c}} \mathrm{PCS}$ and MCS were included in ordinary least squares model

${ }^{\mathrm{d}} \mathrm{PCS}, \mathrm{MCS}$, and the interaction terms were used in multinomial logit model

${ }^{\mathrm{e}}$ Individual SF-12 questions were used in multinomial logit model

\section{Analysis}

In each model, a hypothetical cohort of 10,000 patients was modelled to estimate the incremental costs and QALYs gained from HD and PD for an average patient. Discounting at an annual rate of $3 \%$ was applied to both costs and QALYs. The incremental cost-effectiveness ratios (ICERs) of HD compared to PD were calculated for the two models separately. Difference in EQ-5D and mEQ-5D/SF-6D based incremental QALYs and ICERs was examined. To examine the variability in utility estimates for the two health states in both models, non-parametric bootstrap method was used to estimate the $95 \%$ confidence interval (percentile method) of incremental QALYs and ICERs with 1,000 replications $[27,28]$. All analyses were performed using Microsoft Excel 2016.

\section{Results}

Figure 1 presents the box plots of the utilities values for HD and PD states used in model 1 and model 2. In both models, patients on HD had higher utility values than those on PD. For both HD and PD, mEQ-5D generated lower values than EQ-5D and so did SF-6D. Among the mEQ-5D values, the error margins estimated by the response-mapping functions (i.e. function d. and e.) were wider than the direct-mapping functions. Table 2 summarises the mean utility values and utility differences between HD and PD states. The betweengroup utility differences varied with the approach used to generate utility values; the EQ-5D values exhibited much larger differences than the mEQ-5D and SF-6D values.

Table 2 also presents the estimated incremental QALYs. In model 1, incremental QALY was 2.011 using EQ-5D values, 1.343 to 1.399 using mEQ-5D values, and 1.427 using SF-6D values. The corresponding figures were 1.603, 1.215 to 1.364 , and 1.364 in model 2. Compared to observed EQ-5D values, mapping algorithms generated much lower incremental QALYs (range -14.9 to $-33.2 \%$ ), with smallest differences using SF-12 summary scores to item responses mapping algorithm, i.e. function $\mathrm{d}(-30.8 \%$ for model 1 and $-14.9 \%$ for model 2 ). The incremental QALYs estimated using SF-6D values were $29.0 \%$ lower for model 1 and $14.9 \%$ lower for model 2, compared to those estimated using observed EQ-5D values. The $95 \%$ confidence interval of incremental QALYs confirmed the lower incremental QALYs estimated using mapped EQ-5D and SF-6D than those estimated using observed EQ-5D (Table 2). The estimated ICERs are also summarised in Table 2. In both models, mEQ-5D generated much higher ICERs than observed EQ-5D (model 1, 43.7-49.7\%; model 2, 17.5-25.4\%) and so did SF-6D (model 1, 40.9\%; model 2, 17.5\%). The 95\% confidence interval of ICERs also confirmed the higher estimated ICERs using mEQ-5D and SF-6D, compared to those using observed EQ-5D (Table 2).

\section{Discussion}

This study observed substantially different results in incremental QALYs and ICERs estimated using mapped and directly captured EQ-5D data. Such results are not surprising as mapping technique has been found to introduce additional uncertainty into cost-effectiveness estimates and thus should be treated as a second best option [6]. First, the discrepancies could be explained by the considerable differences in the descriptive system of SF-12 and EQ-5D. Although both instruments are designed to measure some similar dimensions of health, their descriptive systems seem to capture different aspects of these dimensions [13, 29]. It is worth 
Fig. 1 Box plots of utilities for $\mathrm{HD}$ and PD states used in model 1 (a) and model 2 (b)
A

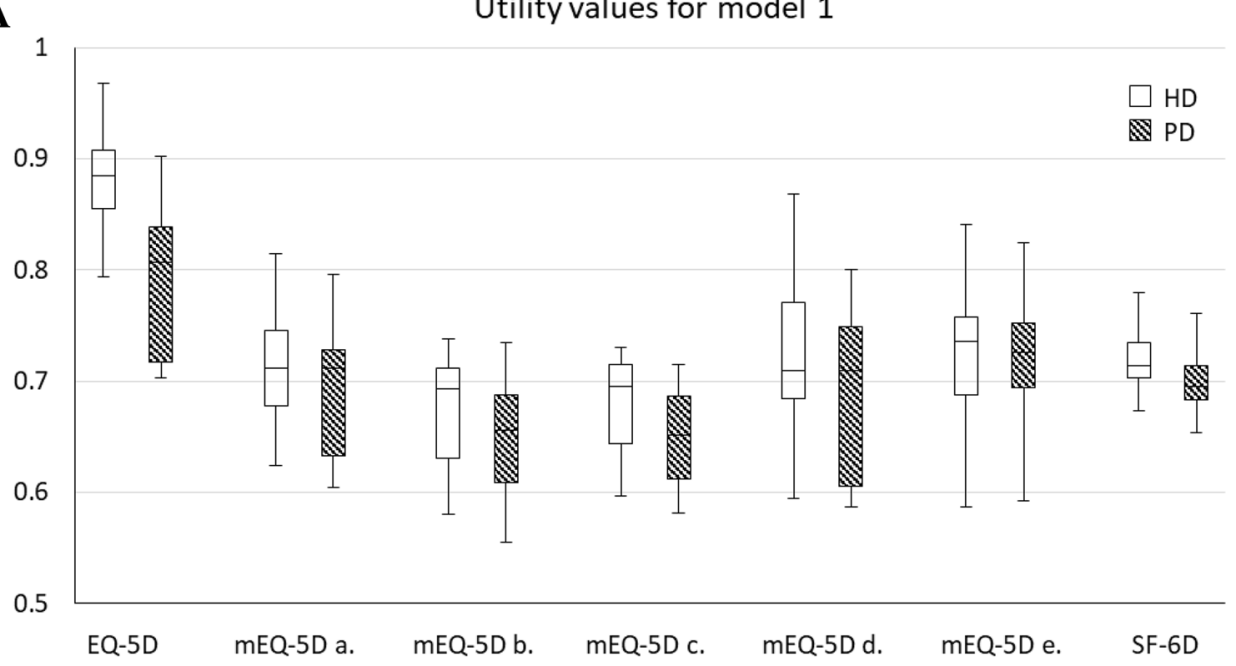

B

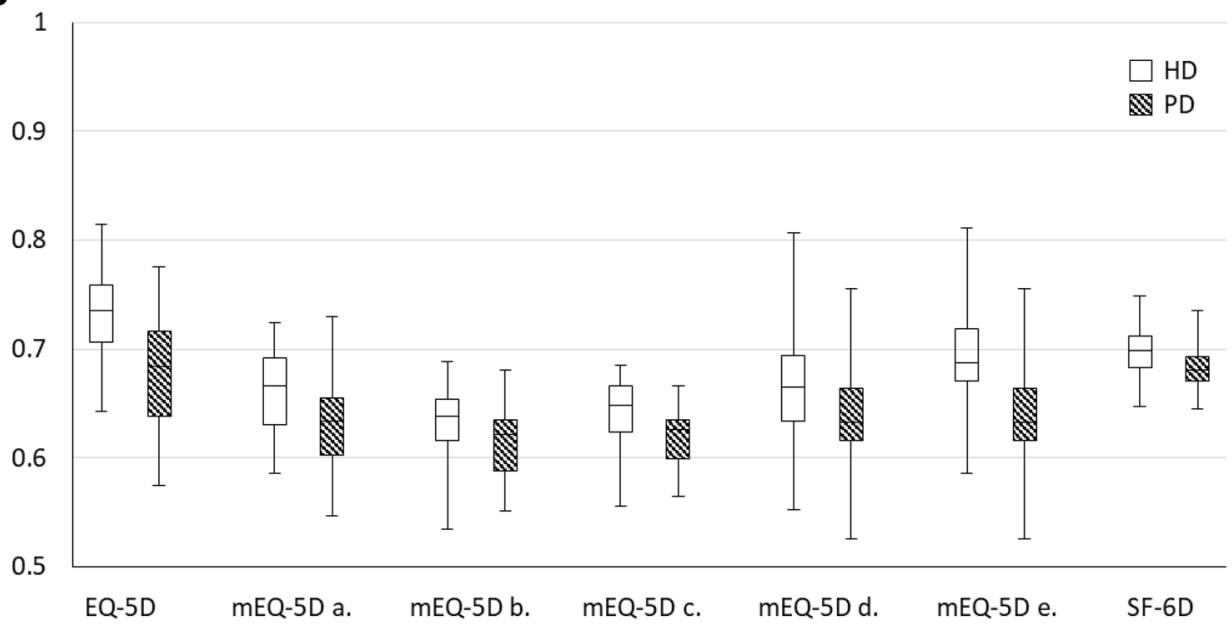

mentioning that the difference in recall period between the two instruments could also matter in this dialysis patient sample. The quality of life data for HD patients were collected while they were undergoing dialysis, and therefore, patients may take the effects of dialysis into consideration when assessing their own health on that day, as measured by EQ-5D, but consider the average health in the past 4 weeks when completing SF-12. As a result, the quality of life for HD patients may be over-estimated if measured using EQ-5D.

Second, the differences may also be due to a mismatch between the mapping functions and the study sample. The validity of mapping is based on the assumption that the statistical relationship is the same between the sample used to develop mapping functions and the target sample to which the mapping functions will be applied [6], so the mapping algorithm developed using data from patients whose characteristics were comparable to this dialysis sample would perform better in terms of validity. However, the currently available mapping functions (and used in this study) were estimated using either a low-income and minority patient sample or general population sample in US [30] which are much younger and healthier than the dialysis patient sample whose data are reported here $[9,11,23]$. The variations in results may imply that the mapping algorithms used in this study are not suitable for this dialysis patient sample. It is ideal to use the mapping functions based on data from dialysis patients, but such algorithm is not available yet.

Third, prediction bias is an inherent weakness of the mapping technique. OLS models used in direct mapping may not accurately predict the EQ-5D distribution for high values due to the ceiling effects of EQ-5D and over-predict utility values for patients in poor health [30]; the response-mapping approach could better reflect the distribution of EQ-5D, but no performance improvement was found [31, 32]. Interestingly, the SF-12 summary scores to EQ-5D response-mapping technique (mapping function $\mathrm{d}$.) seems to perform best among the five algorithms, possibly because the SF-12 and 
Table 2 Mean utility scores, between-group utility differences, incremental QALYs estimated using different methods

\begin{tabular}{|c|c|c|c|c|c|c|c|c|c|}
\hline & \multicolumn{3}{|c|}{ Utilities } & \multicolumn{3}{|c|}{ Incremental QALYs } & \multicolumn{3}{|l|}{ ICER } \\
\hline & HD & PD & $\begin{array}{l}\text { Difference } \\
\text { (HD-PD) }\end{array}$ & Mean & $\%$ Difference & Bootstrap 95\% CI & Mean & $\%$ Difference & Bootstrap 95\% CI \\
\hline \multicolumn{10}{|l|}{ Model 1} \\
\hline EQ-5D & 0.882 & 0.803 & 0.079 & 2.011 & - & $1.891-2.138$ & 59,073 & - & $55,564-62,822$ \\
\hline mEQ-5D a. & 0.714 & 0.701 & 0.013 & 1.384 & $-31.2 \%$ & $1.260-1.519$ & 85,835 & $45.3 \%$ & $78,207-94,283$ \\
\hline mEQ-5D b. & 0.676 & 0.658 & 0.018 & 1.343 & $-33.2 \%$ & $1.230-1.456$ & 88,456 & $49.7 \%$ & $81,591-96,582$ \\
\hline mEQ-5D c. & 0.683 & 0.655 & 0.027 & 1.399 & $-30.4 \%$ & $1.290-1.499$ & 84,915 & $43.7 \%$ & $79,250-92,090$ \\
\hline mEQ-5D d. & 0.721 & 0.709 & 0.012 & 1.392 & $-30.8 \%$ & $1.216-1.564$ & 85,342 & $44.5 \%$ & 75,957-97,694 \\
\hline mEQ-5D e. & 0.725 & 0.723 & 0.002 & 1.348 & $-33.0 \%$ & $1.177-1.511$ & 88,128 & $49.2 \%$ & $78,621-100,931$ \\
\hline SF-6D & 0.718 & 0.698 & 0.020 & 1.427 & $-29.0 \%$ & $1.357-1.488$ & 83,249 & $40.9 \%$ & $79,836-87,543$ \\
\hline \multicolumn{10}{|l|}{ Model 2} \\
\hline EQ-5D & 0.739 & 0.677 & 0.062 & 1.603 & - & $1.490-1.699$ & 70,193 & - & $66,227-75,517$ \\
\hline mEQ-5D a. & 0.661 & 0.631 & 0.030 & 1.342 & $-16.3 \%$ & $1.255-1.425$ & 83,845 & $19.4 \%$ & $78,961-89,657$ \\
\hline mEQ-5D b. & 0.627 & 0.613 & 0.013 & 1.215 & $-24.2 \%$ & $1.123-1.289$ & 92,609 & $31.9 \%$ & $87,292-100,196$ \\
\hline mEQ-5D c. & 0.640 & 0.617 & 0.023 & 1.278 & $-20.3 \%$ & $1.198-1.343$ & 88,044 & $25.4 \%$ & $83,783-93,923$ \\
\hline mEQ-5D d. & 0.671 & 0.640 & 0.031 & 1.364 & $-14.9 \%$ & $1.248-1.501$ & 82,493 & $17.5 \%$ & $74,963-90,160$ \\
\hline mEQ-5D e. & 0.696 & 0.683 & 0.013 & 1.339 & $-16.5 \%$ & $1.241-1.451$ & 84,033 & $19.7 \%$ & $77,547-90,669$ \\
\hline SF-6D & 0.699 & 0.681 & 0.018 & 1.364 & $-14.9 \%$ & $1.315-1.423$ & 82,493 & $17.5 \%$ & $79,072-85,597$ \\
\hline
\end{tabular}

Bootstrap denotes the bootstrap percentile method with 1000 bootstrap replications

$C I$ confidence interval, $H D$ haemodialysis, ICER incremental cost-effectiveness ratio, $P D$ peritoneal dialysis, $E Q-5 D$ EuroQol-5D, $S F-6 D$ Short Form 6-dimension

EQ-5D could be better modelled using summary scores and item responses, respectively.

Last, the use of primary EQ-5D-5L data may also contribute to the discrepancies. The observed EQ-5D values were calculated using the EQ-5D-5L value set while all mapping functions were based on EQ-5D-3L values. Variations in estimating cost effectiveness using $3 \mathrm{~L}$ and $5 \mathrm{~L}$ value sets have been reported previously [33, 34]. However, due to the unavailability of primary EQ-5D-3L data, it is not possible to compare the results of mapped EQ5D-3L values to those observed EQ-5D-3L values.

It should be noted that mapped EQ-5D values consistently generated much lower incremental QALYs and higher ICERs than directly captured EQ-5D values, which could be mainly driven by the much smaller betweengroup utility differences defined by mapped EQ-5D. As shown in a previous study [34] that incremental QALYs based on these two Markov models were a function of both utility of PD and difference in utilities of PD and $\mathrm{HD}$; the performance of mapping algorithms in quantifying absolute utilities of the alternatives also contribute to the different results. The finding that mapped EQ-5D tends to generate fewer incremental QALYs and thus higher ICERs have important implications for decisionmaking in economic evaluation. It is possible that the technologies, which would be considered cost-effective using observed EQ-5D values may be rejected by the reimbursement agency if mapped EQ-5D data were used. Therefore, researchers and decision makers should be aware of the impact of using mapped utility estimates in economic evaluation. It is highly suggested that directly collected EQ-5D data should be used in CUAs to inform decision-making on new or existing health technologies.

When EQ-5D data are not available, but SF-12 data are, estimating health utility using SF-6D could be an alternative for countries without preference for a particular instrument. Our results show that SF-6D generated lower incremental QALY estimates and higher ICERs. A number of differences between SF-6D and EQ-5D could explain the differences, such as differences in the descriptive system [13, 29], valuation techniques (standard gamble used in SF-6D vs. time trade-off used in EQ-5D) $[35,36]$ and value ranges $(0.345$ to 1 in SF-6D vs. -0.594 to 1 in EQ-5D) [25]. This finding suggests that the cost-effectiveness results based on SF-6D are not identical to those based on EQ-5D and if the same willingness-to-pay threshold is applied, the reimbursement decisions based on SF-6D and EQ-5D values may be different. Therefore, for a HTA agency, it is better to designate one single preference-based measure as reference case to achieve consistency in decision-making.

This study is not without limitations. It is based on a single cost-effectiveness analysis of dialysis treatments in the context of Singapore, which undoubtedly limits the generalisability of its findings. Many previous studies including a 
variety of general population and patient samples also found that the smaller between-group utility differences estimated using mapped EQ-5D [37-39], same as this study. Another limitation is that the EQ-5D values were not calculated using the value set derived from the country where the original data were collected. Although the UK value sets were used consistently in both observed and mapped EQ-5D values to minimise the differences resulted from country-specific value sets, the applicability of UK value sets into Singaporean EQ-5D data may still be a concern.

\section{Conclusions}

Compared to observed EQ-5D, mapped EQ-5D and SF-6D, would generate fewer QALY gains and higher ICERs in cost-utility analysis, which may lead to different conclusions about the cost effectiveness of health care. It would be more sensible to conduct CUA studies using directly collected EQ-5D data and to designate one single preference-based measure as reference case in a jurisdiction to achieve consistency in healthcare decision-making.

Acknowledgements This study was partially funded by EuroQol foundation (EQ Project 20170450)

\section{Compliance with ethical standards}

Conflict of interest ND is an employee of the Office of Health Economics, a registered charity, which receives funding from a variety of sources, including the Association of the British Pharmaceutical Industry. ND and NL are members of the EuroQol Group.

Open Access This article is distributed under the terms of the Creative Commons Attribution 4.0 International License (http://creativeco mmons.org/licenses/by/4.0/), which permits unrestricted use, distribution, and reproduction in any medium, provided you give appropriate credit to the original author(s) and the source, provide a link to the Creative Commons license, and indicate if changes were made.

\section{References}

1. Weinstein, M.C., Torrance, G., McGuire, A.: QALYs: the basics. Value Health 12(Suppl 1):5-9 (2009). https://doi.org/10.111 1/j.1524-4733.2009.00515.x

2. NICE: NICE Guide to the methods of technology appraisal 2013. https://www.nice.org.uk/process/pmg9/chapter/the-referencecase\#measuring-and-valuing-health-effects (2013). Accessed 15 Aug 2017

3. CADTH: Guidelines for the Economic Evaluation of Health Technologies: Canada. https://www.cadth.ca/about-cadth/howwe-do-it/methods-and-guidelines/guidelines-for-the-economicevaluation-of-health-technologies-canada (2017). Accessed 12 Dec 2017

4. PBS: Guidelines for preparing submissions to the Pharmaceutical Benefits Advisory Committee (Version 4.2). http://www.pbs. gov.au/info/industry/listing/procedure-guidance/4-presubmiss ion-requirements/4-2-guidelines-for-preparing-submissions (2017). Accessed 12 Dec 2017

5. SMC: Guidance to manufacturers for completion of New Product Assessment Form (NPAF). https://www.scottishmedicines.org. uk/files/submissionprocess/Guidance_on_NPAF_Final_May20 17.doc (2017). Accessed 12 Dec 2017

6. Longworth, L., Rowen, D.: Mapping to obtain EQ-5D utility values for use in NICE health technology assessments. Value Health. 16(1), 202-210 (2013). https://doi.org/10.1016/j.jval.2012.10.010

7. Obradovic, M., Lal, A., Liedgens, H.: Validity and responsiveness of EuroQol-5 dimension (EQ-5D) versus Short Form-6 dimension (SF-6D) questionnaire in chronic pain. Health Qual. Life Outcomes 11, 110 (2013). https://doi.org/10.1186/1477-7525-11-110

8. Doble, B., Lorgelly, P.: Mapping the EORTC QLQ-C30 onto the EQ-5D-3L: assessing the external validity of existing mapping algorithms. Qual. Life Res. 25(4), 891-911 (2016). https://doi. org/10.1007/s11136-015-1116-2

9. Franks, P., Lubetkin, E.I., Gold, M.R., Tancredi, D.J., Jia, H.: Mapping the SF-12 to the EuroQol EQ-5D Index in a national US sample. Med Decis Making 24(3), 247-254 (2004)

10. Fredriksson, T., Pettersson, U.: Severe psoriasis-oral therapy with a new retinoid. Dermatologica 157(4), 238-244 (1978)

11. Gray, A.M., Rivero-Arias, O., Clarke, P.M.: Estimating the association between SF-12 responses and EQ-5D utility values by response mapping. Med. Decis. Mak. 26(1), 18-29 (2006). https ://doi.org/10.1177/0272989X05284108

12. Brazier, J., Roberts, J., Deverill, M.: The estimation of a preference-based measure of health from the SF-36. J. Health Econ. 21(2), 271-292 (2002)

13. Yang, F., Lau, T., Lee, E., Vathsala, A., Chia, K.S., Luo, N.: Comparison of the preference-based EQ-5D-5L and SF-6D in patients with end-stage renal disease (ESRD). Eur. J. Health Econ. (2014). https://doi.org/10.1007/s10198-014-0664-7

14. Conner-Spady, B., Suarez-Almazor, M.E.: Variation in the estimation of quality-adjusted life-years by different preferencebased instruments. Med. Care. 41(7), 791-801 (2003). https:// doi.org/10.1097/01.MLR.0000068537.83456.36

15. Longworth, L., Bryan, S.: An empirical comparison of EQ-5D and SF-6D in liver transplant patients. Health Econ. 12(12), 1061-1067 (2003). https://doi.org/10.1002/hec.787

16. Davis, J.C., Liu-Ambrose, T., Khan, K.M., Robertson, M.C., Marra, C.A.: SF-6D and EQ-5D result in widely divergent incremental cost-effectiveness ratios in a clinical trial of older women: implications for health policy decisions. Osteoporos. Int. 23(7), 1849-1857 (2012). https://doi.org/10.1007/s0019 8-011-1770-3

17. Sach, T.H., Barton, G.R., Jenkinson, C., Doherty, M., Avery, A.J., Muir, K.R.: Comparing cost-utility estimates: does the choice of EQ-5D or SF-6D matter? Med Care. 47(8), 889-894 (2009). https ://doi.org/10.1097/MLR.0b013e3181a39428

18. Yang, F., Lau, T., Luo, N.: Cost-effectiveness of haemodialysis and peritoneal dialysis for patients with end-stage renal disease in Singapore. Nephrology 21(8), 669-677 (2016). https://doi. org/10.1111/nep.12668

19. Yang, F., Lau, T., Lee, E., Vathsala, A., Chia, K.S., Luo, N.: Comparison of the preference-based EQ-5D-5L and SF-6D in patients with end-stage renal disease (ESRD). Eur. J. Health Econ. 16(9), 1019-1026 (2015). https://doi.org/10.1007/s10198-014-0664-7

20. Rabin, R., de Charro, F.: EQ-5D: a measure of health status from the EuroQol Group. Ann. Med. 33(5), 337-343 (2001). doi:https ://doi.org/10.3109/07853890109002087

21. Ware, J. Jr., Kosinski, M., Keller, S.D.: A 12-Item Short-Form Health Survey: construction of scales and preliminary tests of reliability and validity. Med. Care 34(3), 220-233 (1996) 
22. Devlin, N.J., Shah, K.K., Feng, Y., Mulhern, B., van Hout, B.: Valuing health-related quality of life: an EQ-5D-5L value set for England. Health Econ. (2017). https://doi.org/10.1002/hec.3564

23. Franks, P., Lubetkin, E.I., Gold, M.R., Tancredi, D.J.: Mapping the SF-12 to preference-based instruments: convergent validity in a low-income, minority population. Med. Care. 41(11), 12771283 (2003). https://doi.org/10.1097/01.MLR.0000093480.58308 .D8

24. Lawrence, W.F., Fleishman, J.A.: Predicting EuroQoL EQ-5D preference scores from the SF-12 Health Survey in a nationally representative sample. Med. Decis. Mak. 24(2), 160-169 (2004). https://doi.org/10.1177/0272989X04264015

25. Brazier, J.E., Roberts, J.: The estimation of a preference-based measure of health from the SF-12. Med. Care 42(9), 851-859 (2004)

26. Wyld, M., Morton, R.L., Hayen, A., Howard, K., Webster, A.C.: A systematic review and meta-analysis of utility-based quality of life in chronic kidney disease treatments. PLoS Med. 9(9), e1001307 (2012). https://doi.org/10.1371/journal.pmed.1001307

27. Campbell, M.K., Torgerson, D.J.: Bootstrapping: estimating confidence intervals for cost-effectiveness ratios. QJM 92(3), 177-182 (1999)

28. Briggs, A.H., Wonderling, D.E., Mooney, C.Z.: Pulling cost-effectiveness analysis up by its bootstraps: a non-parametric approach to confidence interval estimation. Health Econ. 6(4), 327-340 (1997)

29. Xie, F., Li, S.C., Luo, N., Lo, N.N., Yeo, S.J., Yang, K.Y., Fong, K.Y., Thumboo, J.: Comparison of the EuroQol and short form 6D in Singapore multiethnic Asian knee osteoarthritis patients scheduled for total knee replacement. Arthritis Rheum. 57(6), 1043-1049 (2007). https://doi.org/10.1002/art.22883

30. Brazier, J.E., Yang, Y., Tsuchiya, A., Rowen, D.L.: A review of studies mapping (or cross walking) non-preference based measures of health to generic preference-based measures. Eur. J. Health Econ. 11(2), 215-225 (2010). https://doi.org/10.1007/s 1019 $8-009-0168-z$
31. Rowen, D., Brazier, J., Roberts, J.: Mapping SF-36 onto the EQ-5D index: how reliable is the relationship? Health Qual. Life Outcomes 7, 27 (2009). https://doi.org/10.1186/1477-7525-7-27

32. Chuang, L.H., Kind, P.: Converting the SF-12 into the EQ-5D: an empirical comparison of methodologies. Pharmacoeconomics. 27(6), 491-505 (2009). https://doi.org/10.2165/00019053-20092 7060-00005

33. Hernandez Alava, M., Wailoo, A., Grimm, S., Pudney, S., Gomes, M., Sadique, Z., Meads, D., O'Dwyer, J., Barton, G., Irvine, L.: EQ-5D-5L versus EQ-5D-3L: the impact on cost effectiveness in the United Kingdom. Value Health. 21(1), 49-56 (2018). https:// doi.org/10.1016/j.jval.2017.09.004

34. Yang, F., Devlin, N., Luo, N.: Cost-utility analysis using EQ5D-5L data: does how the utilities are derived matter? Value Health (2018, accepted)

35. Tsuchiya, A., Brazier, J., Roberts, J.: Comparison of valuation methods used to generate the EQ-5D and the SF-6D value sets. J. Health Econ. 25(2), 334-346 (2006). https://doi.org/10.1016/j. jhealeco.2005.09.003

36. Whitehurst, D.G., Norman, R., Brazier, J.E., Viney, R.: Comparison of contemporaneous EQ-5D and SF-6D responses using scoring algorithms derived from similar valuation exercises. Value Health 17(5), 570-577 (2014). https://doi.org/10.1016/j. jval.2014.03.1720

37. Rowen, D., Young, T., Brazier, J., Gaugris, S.: Comparison of generic, condition-specific, and mapped health state utility values for multiple myeloma cancer. Value Health 15(8), 1059-1068 (2012). https://doi.org/10.1016/j.jval.2012.08.2201

38. Versteegh, M.: Impact on the incremental cost-effectiveness ratio of using alternatives to EQ-5D in a Markov model for multiple sclerosis. Pharmacoeconomics 34(11), 1133-1144 (2016). https ://doi.org/10.1007/s40273-016-0421-0

39. Hoyle, C.K., Tabberer, M., Brooks, J.: Mapping the COPD assessment test onto EQ-5D. Value Health 19(4), 469-477 (2016). https ://doi.org/10.1016/j.jval.2016.01.005 